\title{
Autonomous motion planning of a hand-arm robotic system based on captured human-like hand postures
}

\author{
Jan Rosell · Raúl Suárez • Carlos Rosales · \\ Alexander Pérez \\ \begin{tabular}{l}
0 \\
\hline \\
\hline \\
\hline \\
\hline \\
\hline \\
\hline \\
\hline \\
\hline \\
\hline
\end{tabular}
}

\begin{abstract}
The paper deals with the problem of motion planning of anthropomorphic mechanical hands avoiding collisions and trying to mimic real human hand postures. The approach uses the concept of "principal motion directions" to reduce the dimension of the search space in order to obtain results with a compromise between motion optimality and planning complexity (time). Basically, the work includes the following phases: capturing the human hand workspace using a sensorized glove and mapping it to the mechanical hand workspace, reducing the space dimension by looking for the most relevant principal motion directions, and planning the hand movements using a probabilistic roadmap planner. The approach has been implemented for a four finger anthropomorphic mechanical hand (17 joints with 13 independent degrees of freedom) assembled on an industrial robot ( 6 independent degrees of freedom), and experimental examples are included to illustrate its validity.

\section{]}

?

[

(]

[

(
\end{abstract}

J. Rosell $(\varangle)$ · R. Suárez · C. Rosales · A. Pérez

Institute of Industrial and Control Engineering, Technical

University of Catalonia, Barcelona, Spain

e-mail: jan.rosell@upc.edu

C. Rosales

Institut de Robòtica i Informàtica Industrial (IRI), CSIC-UPC,

Barcelona, Spain

A. Pérez

Escuela Colombiana de Ingeniería "Julio Garavito", Bogotá D.C., Colombia
Keywords Motion planning · Grasping · Manipulation · Mechanical hands

\section{Introduction}

Advances in robotics are producing a number of complex devices with a high number of degrees of freedom (DOF), lots of sensors, and sophisticated controllers to assure stability and a good performance. These devices include different types of robots, adapted to different environments and tasks, and among them the most representative instances are the humanoids (Bluethmann et al. 2003). Particular elements of these robots are the anthropomorphic hands, with a number of DOF usually ranging from 12 (four fingers with 3 independent DOF each one) to 25 (five fingers with 4 independent DOF each one plus some DOF in the palm (Peña et al. 2005). Examples of anthropomorphic hands with four fingers are the Utah/MIT Hand (Jacobsen et al. 1984), DIST Hand (Caffaz and Cannata 1998), LMS Hand (Gazeau et al. 2001); DLR Hand (Butterfass et al. 2004) and MA-I Hand (Suárez and Grosch 2005), and examples of hands with five fingers are the Belgrade/USC Hand (Bekey et al. 1990), Anthrobot-2 Hand (Ali et al. 1993), NTU Hand (Lin and Huang 1996), ROBONAUT (Lovchik and Diftler 1999), Gifu Hand (Kawasaki et al. 2002), Shadow Hand (Shadow Robot Company 2003) and Bolonia Hand 3 (Lotti et al. 2005). Good discussions about robot hands have already been presented (Bicchi 2000; Biagiotti et al. 2004).

Despite the advanced features of these mechanical hands, one of the remaining problems in order to obtain a good outcome from them is the autonomous determination of their movements, which are quite complex and non-evident for the human being in the space of generalized coordinates. This problem can be formulated as a well-known motion 
planning problem, but in a very large dimensional space. Thus, some new approaches are still necessary in order to find solutions in a faster way that can be really implemented and used in practice. This paper presents some developments in this line, looking for procedures that allow the autonomous motion planning of a hand-arm system, trying to mimic human hand postures and caring about collisions with the environment as well as between the different parts of the hand and the arm. The approach presented here has been implemented on a real physical system and it is a significant improvement and generalization of the work first presented by Rosell et al. (2009).

\section{Problem statement and solution overview}

Let $\mathcal{C}=\mathcal{C}^{\mathrm{h}} \times \mathcal{C}^{\mathrm{a}}$ be the configuration space of a hand-arm system, where $\mathcal{C}^{\mathrm{h}}$ and $\mathcal{C}^{\mathrm{a}}$ are the configuration spaces of the hand and of the arm, respectively. Then, the dimension of $\mathcal{C}$ is equal to the number of DOF of the hand plus the number of DOF of the arm. The problem to be solved is the following: given an initial hand-arm configuration $\boldsymbol{c}_{\text {ini }} \in \mathcal{C}$ and a final desired one $\boldsymbol{c}_{\text {goal }} \in \mathcal{C}$, which is a grasp or pregrasp configuration, find a collision free path in $\mathcal{C}$ from $\boldsymbol{c}_{\text {ini }}$ to $c_{\text {goal }}$, i.e. a collision free path for the hand-arm system. The proposed approach looks of particular interest for the movements of the hand-arm system once they are relatively close to the goal configuration, where it likely exists a solution with a linear arm movement in $\mathcal{C}^{\text {a }}$.

The dimension of the search space for this problem (i.e. $\mathcal{C}$ ) is relatively large, and therefore conventional solutions require high computational times. In this context, the proposed approach is based on a reduction of the search space dimension, which is done by looking for a representative subspace $\mathcal{S} C^{\mathrm{h}}$ of the hand configuration space $\mathcal{C}^{\mathrm{h}}$, and looking for continuous valid paths in the compound subspace $\mathcal{S} C=\mathcal{S} C^{\mathrm{h}} \times \mathcal{C}^{\mathrm{a}}$. Of course, there may be solutions in $\mathcal{C}$ not included in $\mathcal{S} C$, thus the selection of a proper $\mathcal{S C}$ (i.e. a proper $S C^{\mathrm{h}}$ ) is a relevant step in the proposed approach. On the other hand, if a solution is found in $\mathcal{S} C$, for sure it is valid in $\mathcal{C}$.

The main consideration that supports the reduction of the search space is that the human hand has several joint movements that are not (completely) independent, and therefore there are some joint positions that can be related in some way. A typical example is given by the last two joints of each finger, which in general cannot be moved independently, and, in the same way, some other correlations can be found when the human hand postures are carefully analyzed. These correlations can be extrapolated to the mechanical hand in order to try to mimic human hand postures.

In our work, a number of samples of human hand postures are captured using a sensorized glove and then mapped to the mechanical hand configuration space $\mathcal{C}^{\mathrm{h}}$. The samples in $\mathcal{C}^{\mathrm{h}}$ are analyzed (using a principal component analysis) to find the direction with largest dispersion, which is iteratively repeated considering orthogonal directions until a new base of $\mathcal{C}^{\mathrm{h}}$ is generated. Then, by selecting the first $n$ vectors of this base and properly choosing a bounding box $B^{\mathrm{h}}$ aligned with these vectors and centered in the mean value of the original set of points, a good bounded approximation of the hand workspace in $\mathcal{S} C^{\mathrm{h}}$ is found.

A relevant previous work in this line (Santello et al. 1998) uses an initial set of grasping configurations to find a bidimensional grasp subspace, i.e. a reduction of the grasp space is performed based on a set of hand configurations used to grasp different objects, and the dependencies between the finger joints were called postural synergies. This subspace is used in other works for telemanipulation purposes (Tsoli and Jenkins 2007) and to look for grasping configurations (Ciocarlie and Allen 2009). In this latter case, a set of hand configurations parameterized with a single parameter (even when all the hand joints may change simultaneously) is called an eigengrasp, and the bidimensional subspace is built with two eigengrasps (i.e. two parameters) and used to look for pre-grasp configurations such that, staring from them, secure grasp are obtained closing the fingers until the object is contacted. The approach can be applied considering any number of eigengrasps, i.e. using grasp subspaces of any dimension. One relevant difference between these works (that are specifically oriented to grasp synthesis), and that presented in this paper (oriented to motion planning) is that here the set of hand configurations used to determine the dependencies between the motions of the finger joints is not limited to grasping configurations, instead we use a set of unconstrained configurations trying to cover the whole hand workspace, thus the finger joint dependencies determined in this work do not represent "grasping configurations" but general "hand movements", for this reason we prefer to call them "principal motion directions" instead of "eigengrasps" (note that they represent "motion directions" in the hand configuration space). The formal definition of the "principal motion directions" is given later in Sect. 4.2. Dimensionality reduction techniques have also been used in the selection of grasping forces (Gabiccini and Bicchi 2010) and to synthesize human-like motion in graphic applications (Safonova et al. 2004).

The approach used in this work can be summarized in the following steps:

1. Obtain samples of the mechanical hand configuration space $\mathcal{C}^{\mathrm{h}}$ (13 DOF) by mapping samples of the human hand configuration space obtained using a sensorized glove (22 DOF) (Sect. 4.1).

2. Find a representative subspace $\mathcal{S} C^{\mathrm{h}}$ of the mechanical hand configuration space $\mathcal{C}^{\text {h }}$ using Principal Component Analysis (Sect. 4.2). 
3. Model the free space of the representative subspace $\mathcal{S} C=\mathcal{S} C^{\mathrm{h}} \times \mathcal{C}^{\mathrm{a}}$.

- Generate samples of the hand-arm subspace $\mathcal{S C}$ (Sect. 5.1).

- Define a neighboring and interconnecting condition between any two samples (Sect. 5.2).

4. Build a roadmap and, given an initial and final hand-arm configurations in $\mathcal{C}$ (not necessarily belonging to $\mathcal{S C}$ ), $\boldsymbol{c}_{\text {ini }}$ and $\boldsymbol{c}_{\text {goal }}$ respectively, connect them to the roadmap and use it to find a free path between them (Sect. 5.3).

\section{Experimental set-up}

The experimental set-up used in this work involves: (a) an anthropomorphic mechanical hand, (b) an industrial robot, (c) a sensorized glove, (d) a hand/robot simulator connected with the real elements. The main relevant details about these elements are:

(a) Anthropomorphic mechanical hand. We use the Schunk Anthropomorphic Hand (SAH) (Schunk GmbH \& Co. KG 2006), shown in Fig. 1, which is based on the DLR hand (Butterfass et al. 2004). It has three fingers with four joints plus the thumb with five joints, in all of them the distal (outer) and middle flexion joints are mechanically coupled, thus there are a total of 17 joints with only 13 independent DOF. The extra DOF of the thumb is called the "thumb base joint" (numbered with "0" in Fig. 1), and moves the whole thumb with respect to the palm.

(b) Industrial robot. The hand is assembled on an industrial robot Stäubli TX 90, shown in Fig. 2, equipped with a CS8 controller. It is a six DOF general purpose robot arm.

(c) Sensorized glove. We use a commercial sensorized glove CyberGlove (shown in Fig. 3). It is a fully instrumented glove that provides 22 joint-angle measurements using resistive bendsensing technology, it includes three flexion sensors per finger, four abduction sensors between the fingers, a palm-arc sensor, and two sensors to measure the flexion and the abduction of the wrist.

(d) Hand and robot simulator. The simulator has been developed in our laboratory and allows the visualization of the hand, either alone or installed on the industrial robot (shown in Fig. 4). It is used to visualize the results of the planner before running the plan in the real system. The simulator can also be used to on-line visualize the movements of the mechanical hand associated with the movements of the human operator hand captured with the sensorized glove, as well as the movements of the industrial robot associated with the movements of the human operator wrist, which are captured using a magnetic wrist tracker with six DOF. The simulator includes collision detection capabilities.

The schema of the whole experimental set-up is illustrated in Fig. 5, including the type of connection between the different elements.

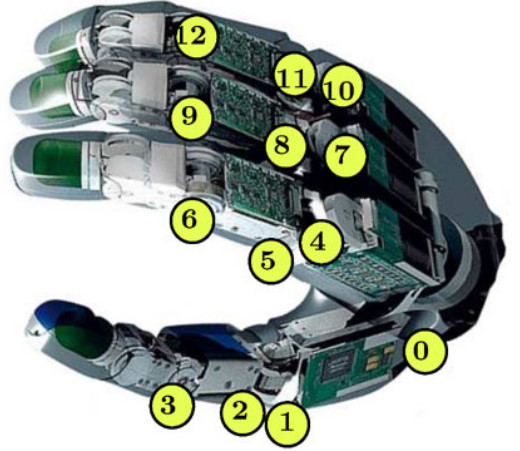

Fig. 1 Anthropomorphic mechanical hand SAH (each number indicates an independent DOF)

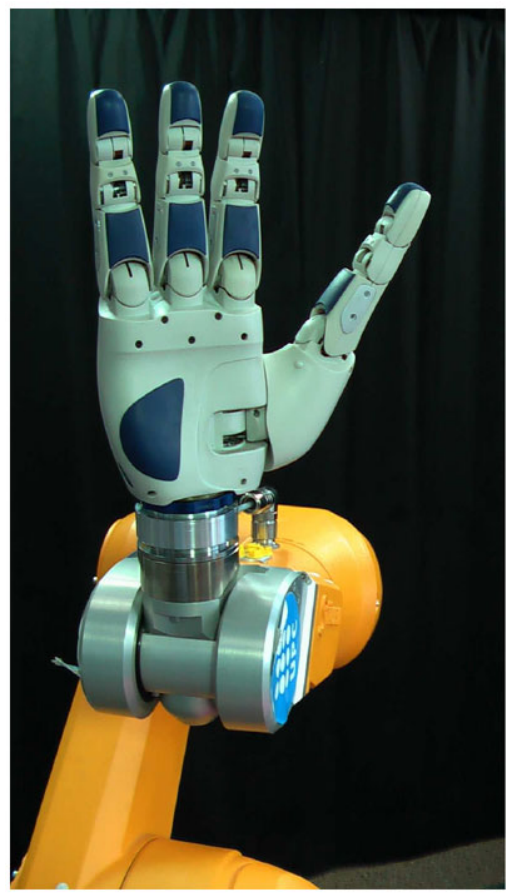

Fig. 2 Industrial robot Stäubli TX 90 with the mechanical hand SAH

\section{Hand postures and principal motion directions}

4.1 Data acquisition and mapping of human hand postures

The postures of a human operator hand are captured using the sensorized glove. The operator freely moves his/her hand in an unconstrained way, i.e. without performing any specific task, trying to cover the whole hand workspace. There is no guarantee that the operator actually covers the whole workspace, but in this way it is expected that he/she performs the most natural and evident hand movements, thus the most natural and evident hand postures are captured. The operator can have a continuous visual feedback of the mechanical hand postures associated with his/her hand postures by means of the hand simulator (Fig. 6). 


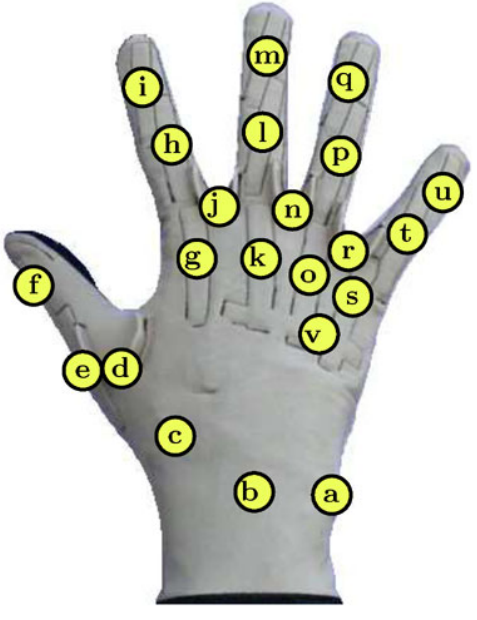

Fig. 3 Sensorized glove used to capture the operator hand workspace (each letter indicates a sensor)

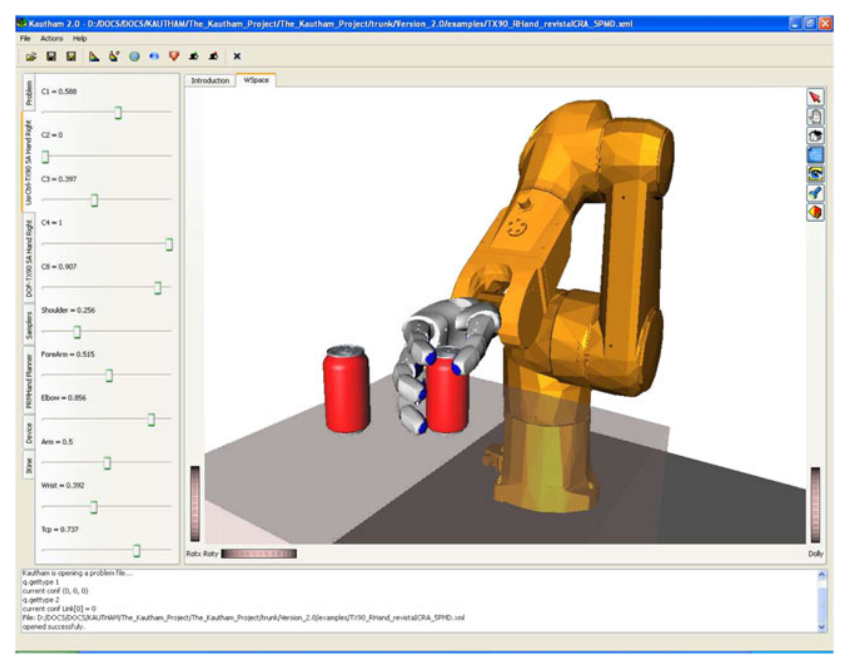

Fig. 4 Hand and robot simulator including the planning environment

In order for the mechanical hand to mimic human hand postures, the mapping of the data obtained from the glove sensors to the joints of the SAH mechanical hand is done considering the following issues (see Figs. 1 and 3):

- The palm of the mechanical hand is rigid, therefore the palm arc sensor $v$ and the wrist flexion and abduction sensors $b$ and $a$ are ignored.

- The mechanical hand lacks the little finger, therefore the sensors $u, t, s$ and $r$ are ignored.

- The mechanical hand has a one-to-one coupling between the medium and distal phalanx of each finger (as in general happen with the human hand), therefore the distal phalanx sensors $i, m$, and $q$ are ignored.

- The abduction is measured in a relative way in the glove, i.e. sensors $j$ and $n$ give, respectively, the relative angle between the index and the middle fingers and between the middle and the ring fingers. Therefore, the mapping is

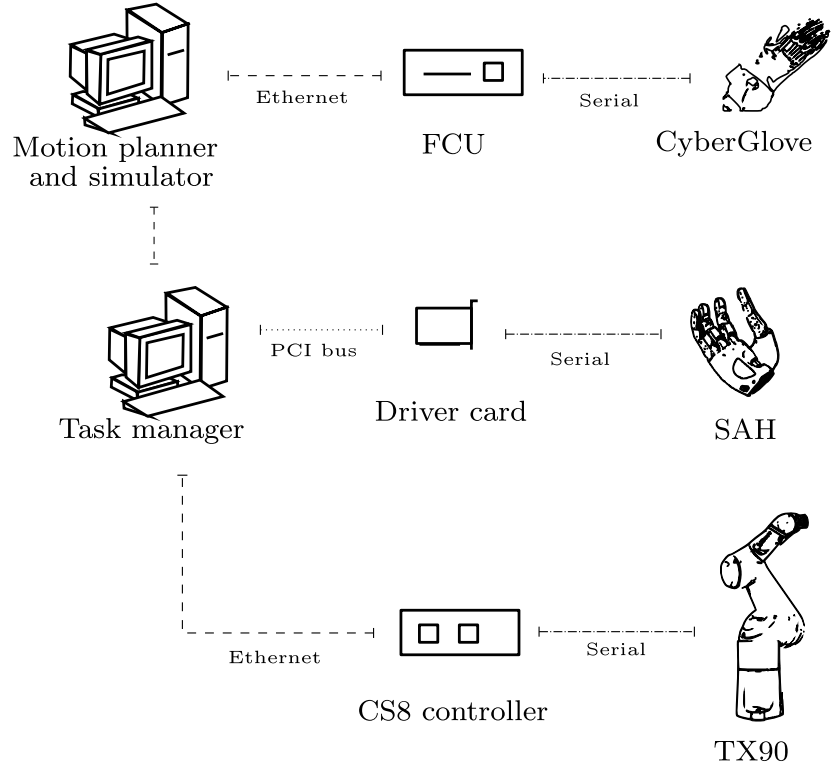

Fig. 5 Schema of the experimental set-up

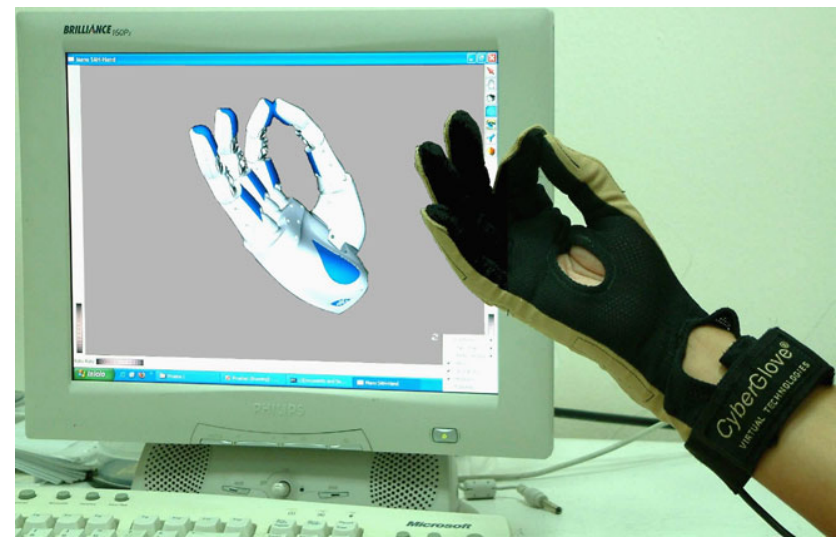

Fig. 6 Human hand with the sensorized glove connected to the mechanical hand simulator

done using the middle finger as reference, i.e. the base of the middle finger (joint 7) is fixed to zero, and sensors $j$ and $n$ are directly associated to joints 4 and 10, respectively.

- The use of sensor $c$ to control joint 1 produces a more natural motion of the SAH hand than using sensor $d$, because sensor $d$ measures the relative abduction between the thumb and the index. Therefore sensor $c$ is used for both joints 0 and 1 .

Then, only 11 values from the 22 sensors available in the glove are used in practice to command the joints of the SAH mechanical hand. The complete mapping is shown in Table 1 . Note that this mapping makes the motions of the SAH hand to be defined with 11 independent parameters despite it has 13 DOF. 
Table 1 Correspondence between the joints of the SAH hand (Fig. 1) and the CyberGlove sensors (Fig. 3)

\begin{tabular}{|c|c|c|c|}
\hline \multicolumn{2}{|c|}{ SA Hand Joint } & \multicolumn{2}{|c|}{ Cyberglove Sensor } \\
\hline Id. & Name & Id. & Name \\
\hline 0 & thumb base & $\mathrm{c}$ & thumb roll \\
\hline 1 & finger base (thumb) & $\mathrm{c}$ & thumb roll \\
\hline 2 & proximal phalanx (thumb) & $\mathrm{e}$ & thumb inner \\
\hline 3 & medium phalanx (thumb) & $\mathrm{f}$ & thumb outer \\
\hline 4 & finger base (index) & $\mathrm{j}$ & index abduction \\
\hline 5 & proximal phalanx (index) & $\mathrm{g}$ & index inner \\
\hline 6 & medium phalanx (index) & $\mathrm{h}$ & index middle \\
\hline 7 & finger base (medium) & - & medium abduction \\
\hline 8 & proximal phalanx (medium) & $\mathrm{k}$ & medium inner \\
\hline 9 & medium phalanx (medium) & 1 & medium medium \\
\hline 10 & finger base (ring) & $\mathrm{n}$ & ring abduction \\
\hline 11 & proximal phalanx (ring) & $\mathrm{O}$ & ring inner \\
\hline 12 & medium phalanx (ring) & $\mathrm{p}$ & ring medium \\
\hline
\end{tabular}

\subsection{Principal motion directions}

Dimensionality reduction of a feature set is a common preprocessing step used for pattern recognition and classification applications as well as in compression schemes. Principal Component Analysis (PCA) is often used in these fields to reduce multidimensional data sets to lower dimensions for their analysis or treatment (Jolliffe 2002), and it is also used as a tool in exploratory data analysis as well as for making predictive models. Basically, PCA involves the computation of the eigenvalue decomposition of a data covariance matrix or the singular value decomposition of a data matrix, usually after mean centering the data for each attribute. The larger the eigenvalues or the singular values the larger the dispersion of the data along the corresponding eigenvector direction. This analysis allows the identification of the directions of the space where the samples have larger dispersion.

In this work, PCA is used to reduce the configuration space $\mathcal{C}^{\text {h }}$ of the mechanical hand SAH to a more tractable space of smaller dimension $\mathcal{S} C^{\mathrm{h}}$, using for that purpose the data obtained from the hand postures of a human operator mapped to the mechanical hand, as described in the previous subsection. The dimension reduction is done based on the correlation that there exists between some joints of the mechanical hand when it follows the hand postures of the human operator. For instance, for a set of 13,500 hand postures captured with the sensorized glove, Fig. 7 shows different examples of the obtained correlations between some particular pairs of joints. ${ }^{1}$

\footnotetext{
${ }^{1}$ The joint values of the SAH hand obtained from the readings of the sensorized glove and the mapping of Table 1 are available at http://iocnet.upc.edu/usuaris/RaulSuarez/proyectos/proa/PROAMiscellanea.html.
}

From the captured data it can be seen that the position of joint 0 of the mechanical hand (the thumb base) is rather independent of the other hand joints (of course, with exception of joint 1 that is completely equivalent due to the selected mapping); two examples are given in the bottom row of Fig. 7. This, together with the fact that joint 0 moves the whole thumb with respect to the palm and therefore changes qualitatively the set of postures the hand can achieve, motivates the selection of joint 0 to form part of a base of $\mathcal{S} C^{\mathrm{h}}$. The remaining directions of the base of $\mathcal{S} C^{\mathrm{h}}$ are obtained applying PCA to the samples of the mechanical hand. PCA returns a new base of the configuration space $\mathcal{C}^{\mathrm{h}}$, with the base vectors ordered according to the dispersion of the samples along each vector direction (the first vector indicates the direction of maximal dispersion of the samples). The directions indicated by these vectors in $\mathcal{C}^{\mathrm{h}}$ are called Principal Motion Directions (PMDs). In order to illustrate the variation of the hand configuration along the PMDs, Fig. 8 shows the hand postures along the two first PMDs, and Fig. 9 the postures resulting from their linear combination.

In our experimental dataset, the first PMD represents the $42.19 \%$ of the total variance, the first two components the $77.12 \%$, and the first three components the $84.71 \%$. The total accumulated variance as a function of the number of selected first PMDs is shown in Fig. 10. Following this result, in this work the use of up to four PMDs has been considered enough to represent, together with the thumb base, the desired subspace $\mathcal{S} C^{\mathrm{h}}$ of $\mathcal{C}^{\mathrm{h}}$. Therefore, the search subspace $\mathcal{S} C^{\mathrm{h}}$ is of dimension up to 5 , defined by the position of the thumb base (joint 0 of the mechanical hand) plus up to 4 PMDs obtained from the samples of hand postures. Note that the inclusion of the thumb base to define one of the dimensions of $\mathcal{S} C^{\mathrm{h}}$ is a particularity related with the use of the mechanical hand SAH and it does not reduce the generality of the approach, which can be applied in a general way just considering $\mathcal{S} C^{\mathrm{h}}$ to be defined by the desired number of PMDs.

\section{Motion planning}

The search of a collision-free path to move a robot from an initial to a goal configuration can be performed in different ways (Choset et al. 2005), being the sampling-based approaches the best alternative for high DOF problems. These approaches outperform other planners because they avoid the explicit characterization of the obstacles in the configuration space $\mathcal{C}$, which is a complex issue even when it is done in an approximate way for a general single-chain revolute manipulator in a polyhedric environment (Lozano-Perez 1987). Sampling-based approaches rely on the generation of collision-free samples of $\mathcal{C}$, in order to capture the connectivity of the free space by connecting the samples with free 

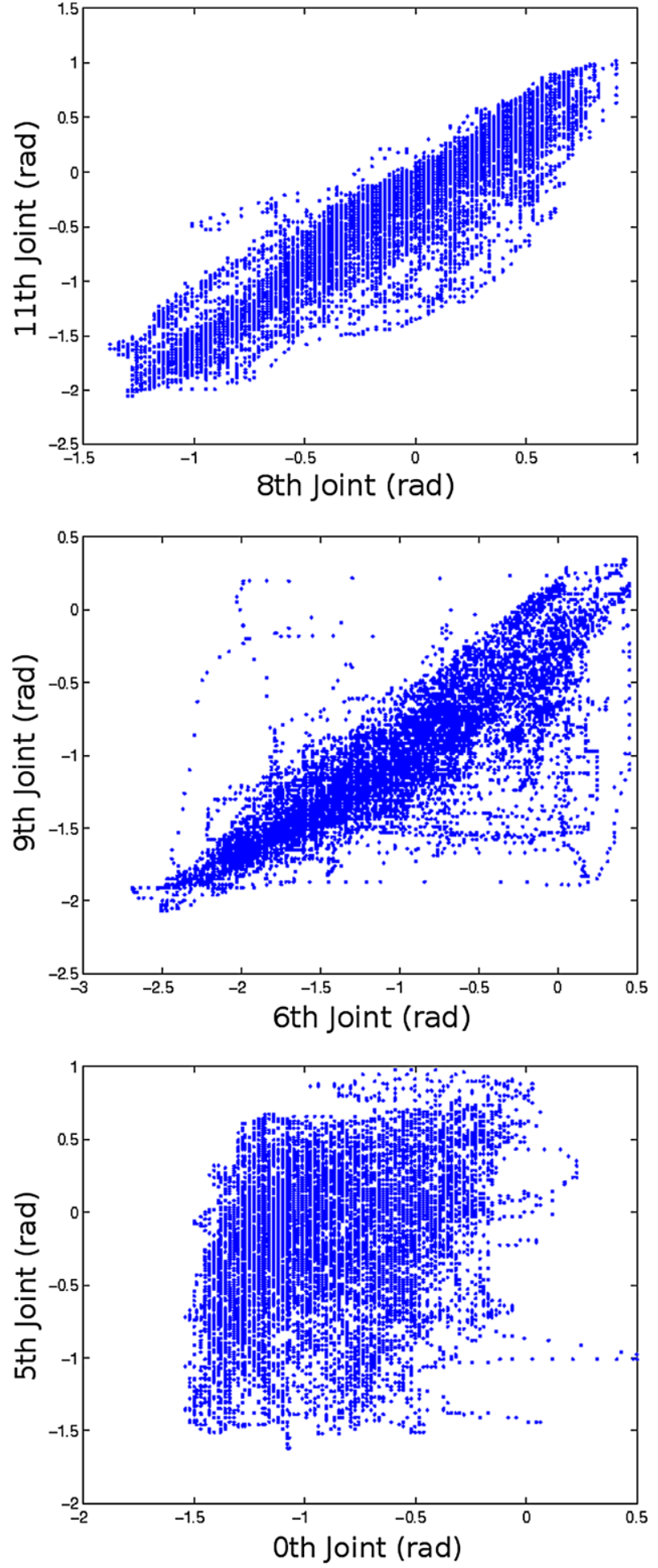

Fig. 7 Top-left: Positive correlation between proximal phalanxes (joints 8 and 11); Top-right: Negative correlation between the index and the ring abductions/adductions (joints 4 and 10); Center-left: Positive correlation between two medium phalanxes (joints 6 and 9); Center-right: Positive correlation between a medium phalanx and an
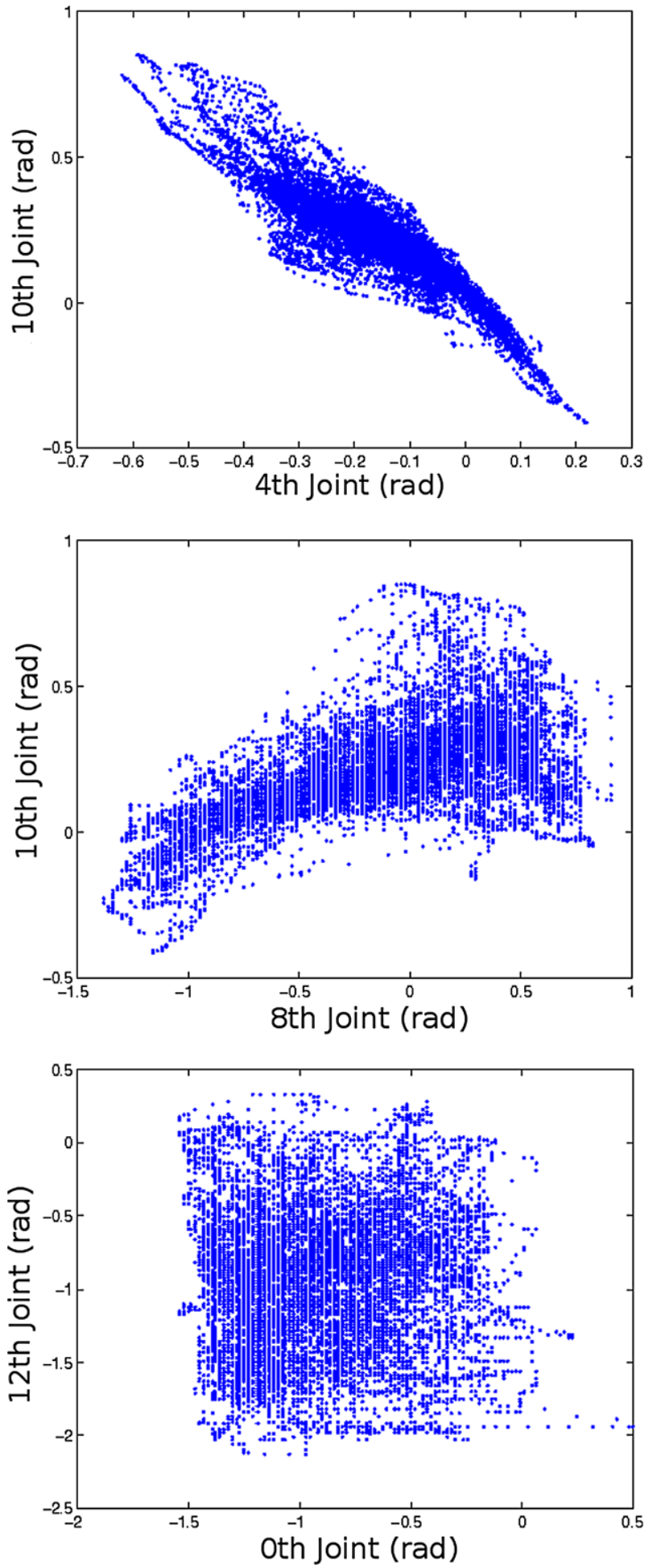

abduction/adduction movement (joints 8 and 10); Bottom-left: No correlation between the thumb base and the medium phalanx of the index (joints 0 and 5); Bottom-right: No correlation between the thumb base and the medium phalanx of the ring finger (joints 0 and 12) 
Fig. 8 Configurations of the SAH hand when it is moved along the first two PMDs

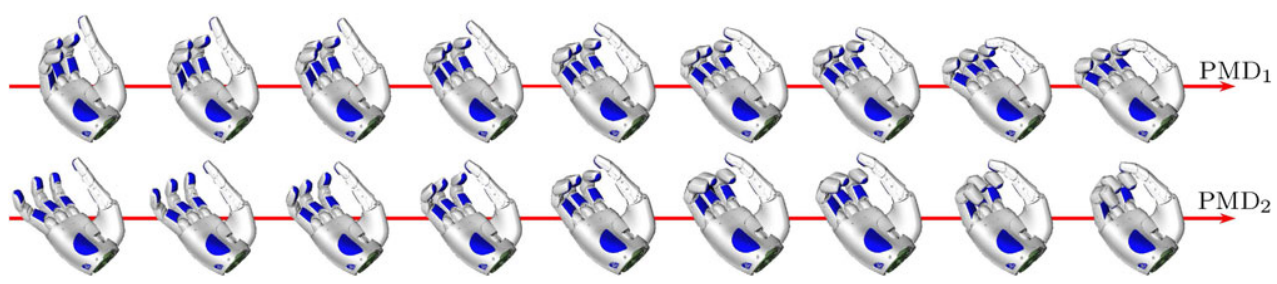

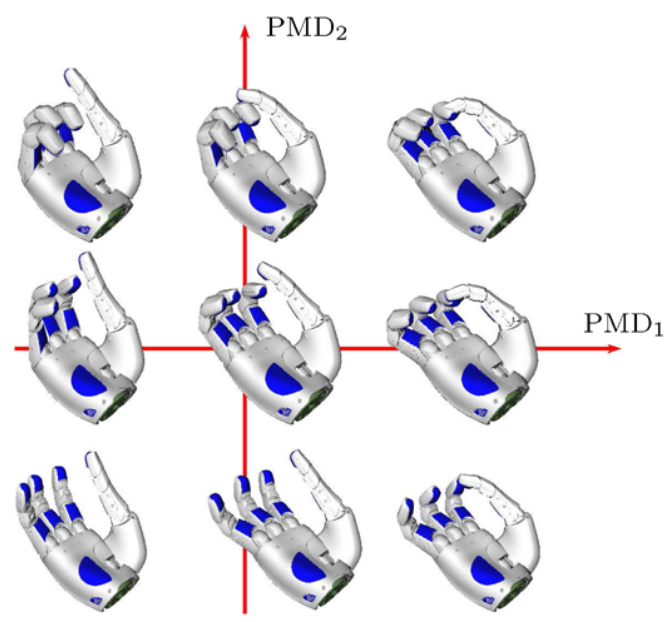

Fig. 9 Configurations of the SAH hand when it is moved along a combination of the first two PMDs

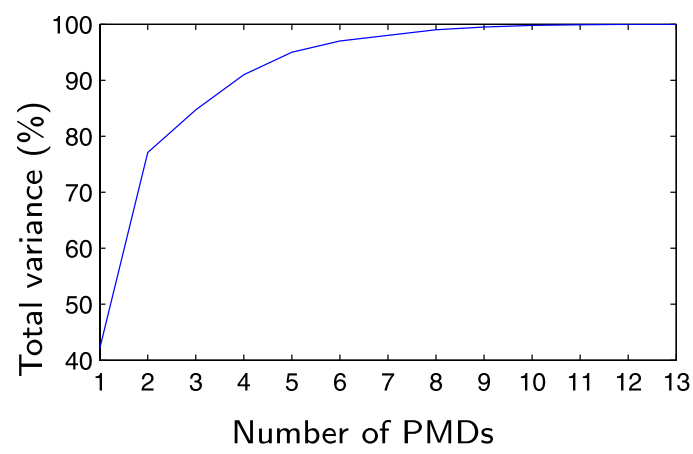

Fig. 10 Total variance covered when using an increasing number of PMDs

paths forming either roadmaps (Kavraki and Latombe 1994) or trees (Kuffner and LaValle 2000). These approaches are demonstrated to be probabilistic complete, and the key issue in their performance is the ability to generate samples in those areas of $\mathcal{C}$ relevant to the problem, either by using importance sampling or dimension-reduction techniques (Geraerts and Overmars 2006).

Some importance sampling strategies increase the density of samples in critical areas of $\mathcal{C}$ by using workspace information (e.g. van der Berg and Overmars 2005; Kurniawati and Hsu 2006) or information gathered during the construction of the roadmap or tree (e.g. Kavraki et al. 1996; Hsu et al. 2005). Others over-sample $\mathcal{C}$ and then filter non- promising configurations (e.g. Boor et al. 1999; Hsu et al. 2003), or deform (dilate) the free regions of $\mathcal{C}$ to make it more expansive and capture its connectivity more easily (e.g. Saha et al. 2005; Cheng et al. 2006). A more detailed discussion of these strategies is given by Hsu et al. (2006). Dimension-reduction techniques, on the other hand, focus on defining the submanifolds of $\mathcal{C}$ where the solution lies (or where a solution is more easily found), and where samples are to be obtained, like for instance submanifolds defined by those configurations that satisfy kinematic closure constraints (Cortés and Siméon 2004), dynamic constraints (Kuffner et al. 2002), or a given set of task-dependant geometric constraints (Berenson et al. 2009; Murrieta-Cid et al. 2005; Rodríguez et al. 2009; Stilman 2010).

In this work, we present a new approach for the motion planning of an anthropomorphic hand assembled on a robot arm. It is of particular interest for the hand-arm movement close to the goal configuration, i.e. when the existence of a free path for the arm considering a bounding volume for the hand is unlikely to be found, and therefore the movements must be planned in the high dimensional space defined by the hand-arm degrees of freedom. Berenson et al. (2007) presented a related work in this line, however it focuses on the developing of a cost function that takes into consideration the surroundings of the object to be grasped and the reachability of the manipulator when the goal grasping configuration is selected, while the planning is done using the high-dimensional configuration space. Here, the goal configuration is provided by grasp synthesis algorithms (Rosales et al. 2011; Rosell et al. 2005), and the contribution relies in the efficiency of the motion planning algorithm, which is done by sampling hand configurations from lower dimensional subspaces defined by subsets of PMDs, and simultaneously sampling arm configurations around the segment that connects the initial and the goal arm configurations. Note that, both the initial and final configurations do not necessarily belong to the lower dimensional subspace since it is not an objective in those previous works. Though, the PMDs are embedded in the configuration space as described in Sect. 2, and thus, the initial and final configuration are neighbors to the samples generated using the PMDs and their interconnection can be done in that space. A different approach to solve this issue when the interconnection is done in the lower dimensional space was proposed in Suárez 


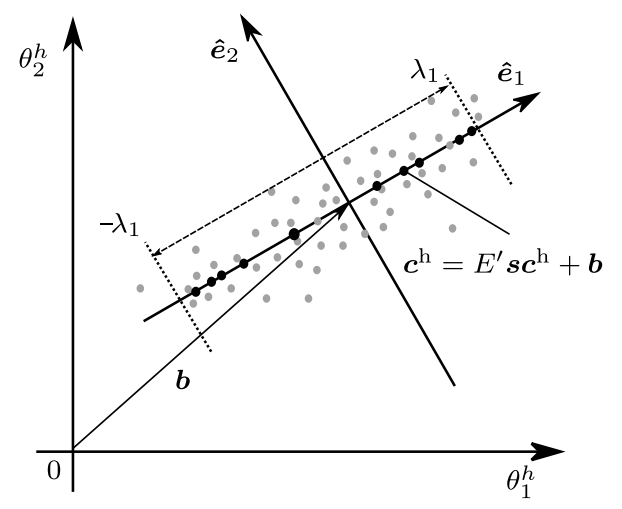

Fig. 11 A 2-dimensional space $\mathcal{C}^{\text {h }}$ modelled with two PMDs, $\hat{\boldsymbol{e}}_{1}$ and $\hat{\boldsymbol{e}}_{2}$, obtained from the input dataset (gray points). The subspace $\mathcal{S} C^{\mathrm{h}}$ is 1-dimensional and defined by $E^{\prime}=\left(\hat{\boldsymbol{e}}_{1}\right)$. Samples (big black dots on the $\hat{\boldsymbol{e}}_{1}$-axis) are obtained from the sampling box $B^{\mathrm{h}}$ that in this case is the segment $\left[-\lambda_{1}, \lambda_{1}\right]$

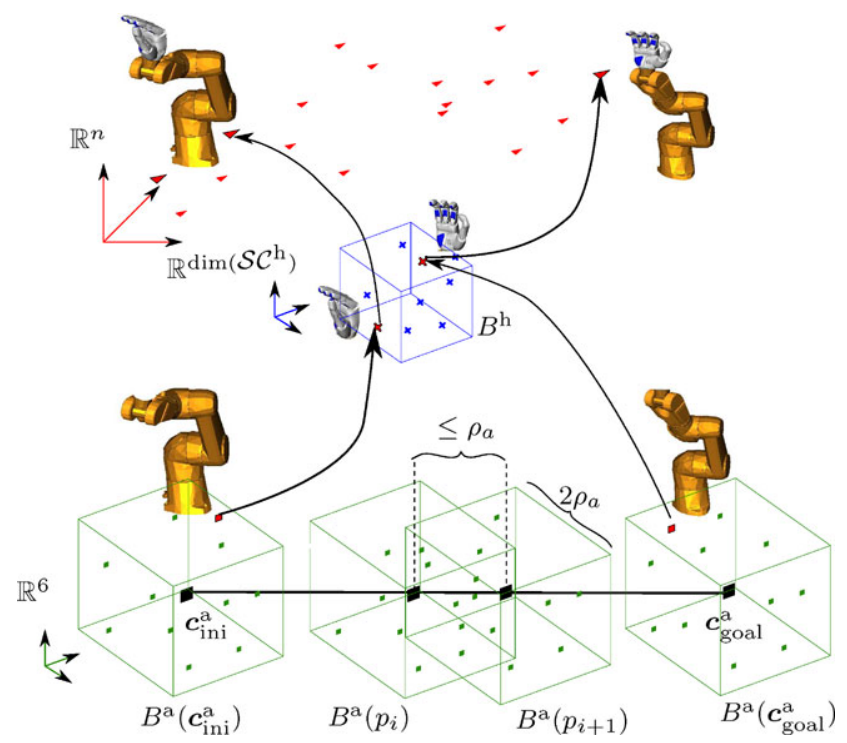

Fig. 12 Samples of the hand-arm system as a composition of arm configurations and hand configurations

et al. (2009), where the initial and final configurations are used to define an additional principal motion direction, such that they belong to the lower dimensional space by construction. The following subsections detail the sampling issues and the proposed general planning algorithm.

\subsection{Sample generation}

The basic features of the procedure to sample hand-arm configurations are listed below, and then the sampling algorithms are formally presented. The features are the following:

1. A random sampling source is considered.

2. Hand configurations are sampled from $B^{\mathrm{h}}$, an axisaligned box in $\mathcal{S} C^{\mathrm{h}}$, with each box side, $2 \lambda_{i}$, proportional to the deviation of the data set in the corresponding principal motion direction. Let:

- $E^{\prime}=\left(\hat{\boldsymbol{e}}_{1}, \ldots, \hat{\boldsymbol{e}}_{H}\right)$ be a matrix with a base of $\mathcal{S} C^{\mathrm{h}}$ as columns,

- $\boldsymbol{s} \boldsymbol{c}^{\mathrm{h}}=\left(\boldsymbol{e}_{1}, \ldots, \boldsymbol{e}_{H}\right)$ with $\boldsymbol{e}_{i} \in\left[-\lambda_{i}, \lambda_{i}\right]$ be a sample obtained with uniform sampling inside $B^{\mathrm{h}}$,

- $\boldsymbol{b}$ be the mean value of the data set used for the PCA analysis.

Then, the joint values $\boldsymbol{c}^{\mathrm{h}}$ of the hand are obtained as (Fig. 11):

$c^{\mathrm{h}}=E^{\prime} \boldsymbol{s} \boldsymbol{c}^{\mathrm{h}}+\boldsymbol{b}$

In the present work, the dimension of $\mathcal{S} C^{\mathrm{h}}$ is not a fixed parameter but a parameter that is iteratively increased by the planning algorithm, as required by the task. Correspondingly, the number of columns of $E^{\prime}$ is iteratively increased, starting with two: the first corresponding to the motion of the thumb-base and the second to the motion defined by the first PMD.

3. A sampling region for the arm configurations is defined around the segment $s_{a}$ that connects $\boldsymbol{c}_{\text {ini }}^{\mathrm{a}}$ and $\boldsymbol{c}_{\mathrm{goal}}^{\mathrm{a}}$, the initial and the goal arm configurations. This region is defined as the union of hypercubes, $B^{\mathrm{a}}\left(p_{i}\right)$, of side $2 \rho_{a}$ centered at evenly spaced points $p_{i} \in s_{a}$ separated a distance $d \leq \rho_{a}$ (Fig. 12). The order in which the hypercubes are swept follows the Van der Corput sequence (Kuipers and Niederreiter 2005), i.e. considering $s_{a}$ of unitary length, points $p_{a}$ are located along $s_{a}$ at the following distances from $c_{\text {ini }}^{\mathrm{a}}: 0,1,0.5,0.25,0.75,0.125$, ....

4. To obtain a collision-free hand-arm configuration, an arm configuration is sampled from each hypercube $B^{\mathrm{a}}\left(p_{i}\right)$ and a hand configuration sampled from $B^{\mathrm{h}}$ is associated to it, until a non-collision hand-arm configuration is found. This is done trying up to $n_{A}$ arm configurations and for each of them up to $n_{H}$ hand configurations, using each time an increasing number of PMDs.

Algorithms 1 and 2 detail, respectively, the sampling procedures for the arm and the hand. They are called from the main algorithm (detailed later in Sect. 5.3) that has the values $n_{H}$ and $n_{A}$ as fixed input parameters, and $\rho_{a}$ as an input parameter that takes increasing values. The following functions are used in Algorithms 1 and 2:

- $\operatorname{RAND}(k,[a, b]):$ Returns a vector of dimension $k$ whose components have random values in the range $[a, b]$.

- SELFCOLLISION $(c)$ : Takes as a parameter either a hand-arm configuration or an arm configuration. In the former case the function returns true if $c$ makes the hand-arm system to be in self-collision, or false otherwise. In the later case the function returns true if $c$ makes the arm to be in selfcollision, or false otherwise. 

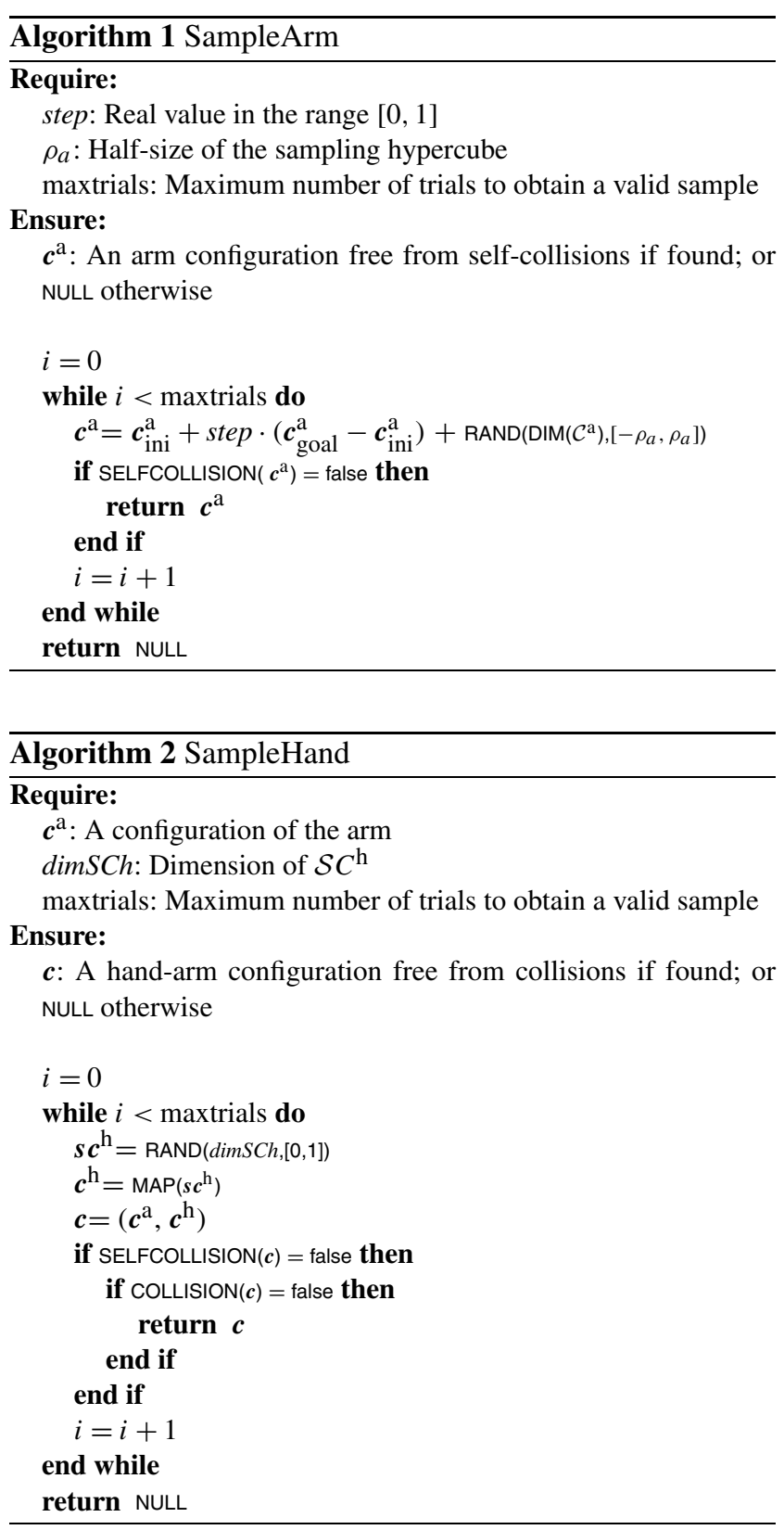

- Collision $(c)$ : Returns true if the input configuration $c \in \mathcal{C}$ makes the hand-arm system to be in collision with the environment, or false otherwise.

- DIM $(S)$ : Returns the dimension of the space $S$.

- $\operatorname{MAP}\left(s c^{\mathrm{h}}\right)$ : Returns the configuration $c^{\mathrm{h}} \in \mathcal{C}^{\mathrm{h}}$ corresponding to $s c^{\mathrm{h}} \in \mathcal{S} C^{\mathrm{h}}$, as computed by (1).

\subsection{Sample interconnection}

The main features of the interconnection procedure are the following:

1. The maximum number of neighboring samples is limited to the closest $K$ samples, being $K$ a predefined value.

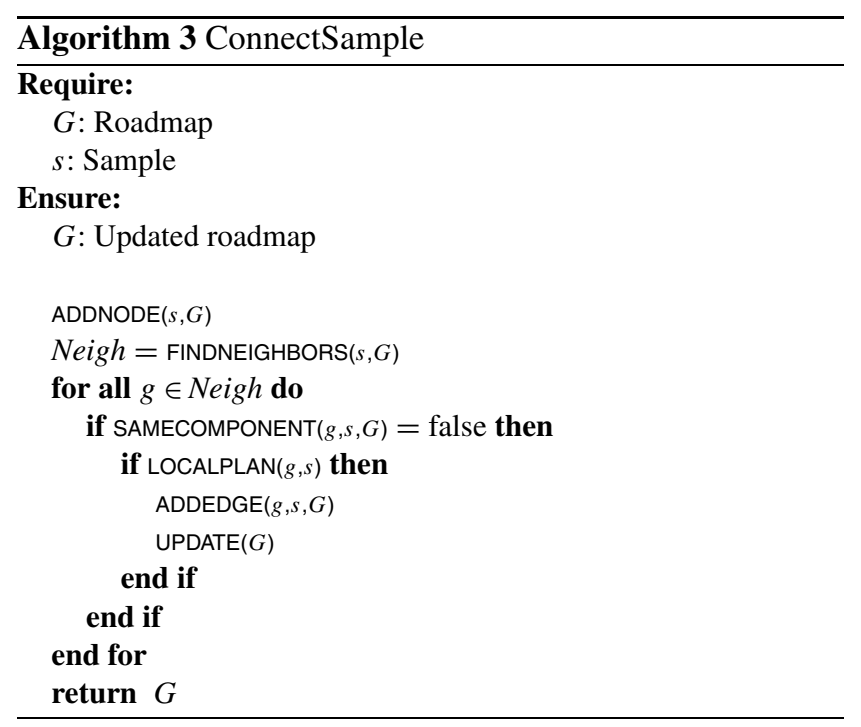

2. All the samples generated within the hypercube centered at $c_{\text {ini }}^{\mathrm{a}}, B^{\mathrm{a}}\left(\boldsymbol{c}_{\text {ini }}^{\mathrm{a}}\right)$, are forced to have $\boldsymbol{c}_{\text {ini }}$ as a neighboring configuration, irrespective of whether $c_{\text {ini }}^{\mathrm{a}}$ belongs to the closest $K$ neighbors or not. The same is done for the goal configuration $c_{\text {goal }}^{\mathrm{a}}$.

Algorithm 3 shows the procedure that performs the connection of a sample to the roadmap. The following functions are used in this algorithm:

- FIndNeIghbors $(s, G)$ : Finds the $K$-nearest neighbors of $s$ from all the nodes of the roadmap $G$. The neighboring threshold is set equal to the distance between $\boldsymbol{c}_{\text {ini }}$ and $c_{\text {goal }}$.

- LOCALPLAN $(g, s)$ : Returns true if the rectilinear path connecting $g$ and $s$ is collision-free, or false otherwise. The test is done by collision-checking configurations sampled along the path following the Van der Corput sequence and verifying that all of them are collision-free. The discretization is small enough not to miss any obstacle in the environment.

- ADdnode $(s, G)$ : Adds node $s$ to graph $G$.

- AdDedge $(s, r, G)$ : Adds edge $(s, r)$ to graph $G$.

- SAmecomponent $(s, q, G)$ : Returns true if nodes $s$ and $q$ belong to the same connected component of the graph $G$, or false otherwise.

- UPdATE $(G)$ : Updates the connected components of graph $G$.

\subsection{Main algorithm}

The main algorithm is a probabilistic roadmap planner that samples and interconnects the configurations as detailed in the previous sections. It is an easy-to-tune adaptive algorithm whose principal features are:

1. The dimension of the hand search space $\mathcal{S} C^{\mathrm{h}}$ is iteratively increased when no collision-free hand-arm configurations is found for a given arm configuration in $\mathcal{C}^{\text {a }}$, i.e. 


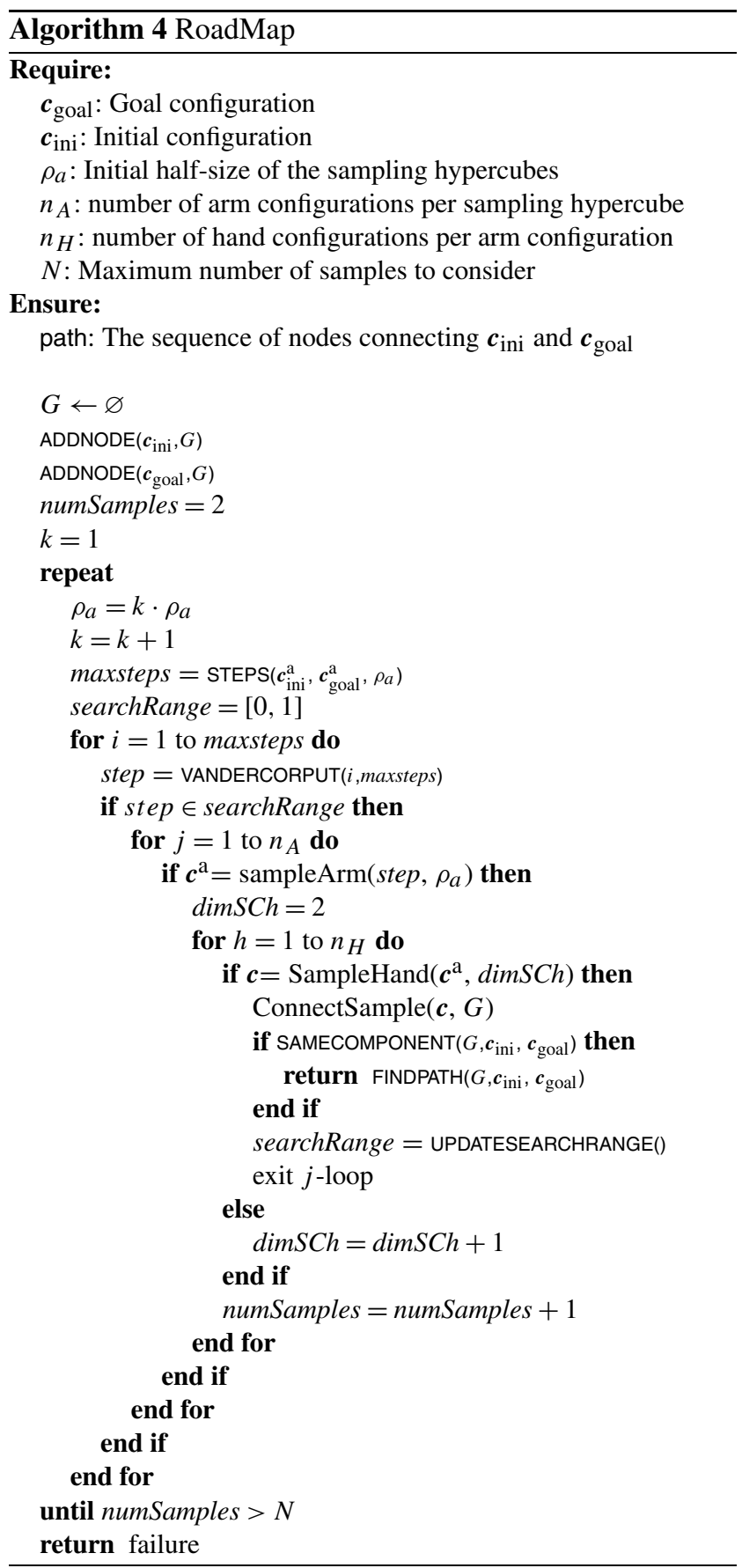

for difficult regions of the configuration space $\mathcal{C}$ more complex hand postures are successively tried.

2. The volume of the arm search space is iteratively increased each time the attempt to connect the initial and the goal configurations fails, i.e. if no solution is found by sampling all the hypercubes $B^{\text {a }}$ (Fig. 12), their size is increased and a new iteration of the algorithm is launched.

3. The main algorithm keeps track of the connected components that contain $\boldsymbol{c}_{\text {ini }}$ and $\boldsymbol{c}_{\text {goal }}$ in order to explore only a subset of the hypercubes $B^{\mathrm{a}}\left(p_{i}\right)$ that define the sam-

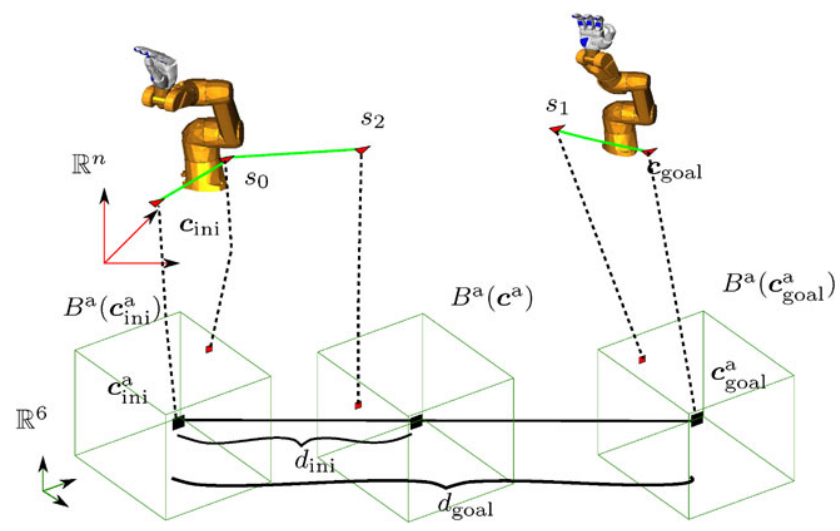

Fig. 13 Example of a roadmap under construction to illustrate the update of the search range $\left[d_{\text {ini }}, d_{\text {goal }}\right]$. Initially $d \in\left[d_{\text {ini }}, d_{\text {goal }}\right]=[0.0,1.0]$ but after having sampled three configurations there are two connected components and the range is $[0.5,1.0]$ : (a) $d_{\text {ini }}$ equals 0.5 since $s_{2}$ is the configuration of the same connected component as $\boldsymbol{c}_{\text {ini }}$ that is obtained from the farthest hypercube, $B^{\mathrm{a}}\left(\boldsymbol{c}^{\mathrm{a}}\right)$, with $c^{\mathrm{a}}$ located at a distance 0.5 from $c_{\text {ini }}^{\mathrm{a}}$; (b) $d_{\text {goal }}$ remains unchanged since $s_{1}$, the unique configuration connected to $c_{\text {goal }}$ was obtained from $B^{\mathrm{a}}\left(c_{\text {goal }}^{\mathrm{a}}\right)$. The update makes that further exploration of the sampling region for the arm configurations be constrained to the hypercubes $B^{\mathrm{a}}$ located at a distance $d \in\left[d_{\text {ini }}, d_{\text {goal }}\right]=[0.5,1.0]$ from $c_{\text {ini }}^{\text {a }}$

pling region for the arm configurations. This is done as follows. Let (Fig. 13):

- $d_{\text {ini }}$ be the maximum distance from $\boldsymbol{c}_{\text {ini }}^{\mathrm{a}}$ to the center of a hypercube that has generated a sample that pertains to the same connected component as $\boldsymbol{c}_{\mathrm{ini}}^{\mathrm{a}}$,

- $d_{\text {goal }}$ be the minimum distance from $\boldsymbol{c}_{\text {ini }}^{\mathrm{a}}$ to the center of a hypercube that has generated a sample that pertains to the same connected component as $\boldsymbol{c}_{\mathrm{goal}}^{\mathrm{a}}$.

Then, only those hypercubes centered at points located at a distance $d \in\left[d_{\text {ini }}, d_{\text {goal }}\right]$ from $\boldsymbol{c}_{\text {ini }}^{\text {a }}$ are likely to generate samples that aid to interconnect the connected component of $\boldsymbol{c}_{\text {ini }}$ with that of $\boldsymbol{c}_{\text {goal }}$, as illustrated in Fig. 13 (take into account that the distance from $\boldsymbol{c}_{\text {ini }}^{\mathrm{a}}$ to $\boldsymbol{c}_{\text {goal }}^{\mathrm{a}}$ is considered unitary and that the hypercubes $B^{\mathrm{a}}$ are swept following the Van der Corput sequence as explained in Sect. 5.1).

4. There are no critical parameters to be tuned, as discussed in detail in Sect. 7.

Algorithm 4 formally details the planning procedure that returns a path connecting $\boldsymbol{c}_{\text {ini }}$ and $\boldsymbol{c}_{\text {goal }}$. The following functions are used:

- STEPS $\left(\boldsymbol{c}_{i}, \boldsymbol{c}_{j}, \rho\right)$ : Computes the number of points evenly spaced along the segment defined by $\boldsymbol{c}_{i}$ and $\boldsymbol{c}_{j}$, such that this number is a power of two and that the distance between two consecutive points is below the given threshold $\rho$. 
- VANDERCORPut $(i$, max $)$ : Computes the value of the $i$ th element in the Van der Corput sequence of max elements, with $\max$ a power of two.

- UPDATESEARCHRANGE(): Updates the range $\left[d_{\text {ini }}, d_{\text {goal }}\right]$.

- FINDPATH $(G, s, q)$ : Returns a path in graph $G$ connecting nodes $s$ and $q$ using the $A^{*}$ algorithm. Once a solution path is found it is smoothed by solving a new (small) roadmap composed of the nodes of the path and all collision-free edges between them.

\section{Experimental validation}

The validation of the proposed approach has been carried out both in a virtual environment with simulated elements, as well as in a real scene with the actual hand-arm system.

\subsection{Implementation issues}

A robot simulation toolkit for motion planning and teleoperation guiding has been developed and it is used to generate and validate the paths before executing them on the physical devices. For the simulator development, three guidelines were considered (Pérez and Rosell 2009): ability to run on different platforms, code accessability and software modularity. The first two led to the use of cross-platform and open-source tools such as Qt for the user interface, Coin3D for the 3D rendering, $\mathrm{PQP}$ for the collision detection, and Boost Graph for the graph management. Regarding the software modularity, the project was conceived to be library-based, thus, different libraries have been developed such as a Geometric library for the treatment of the bodies and their kinematic relation, a Sampling library with different sampling strategies, e.g. Random, Halton (Halton 1960), SDK (Rosell et al. 2007), a Planning library essentially composed of sampling-based planners, e.g. the approach proposed in this work, a Device library for the communication with different devices such as sensorized gloves, robot hands and arms and haptic devices, and, finally the GUI library that implements the user interface and library management.

\subsection{Evaluation of the use of PMDs}

As a benchmark, the task of grasping a can on a table is suggested. The final desired configuration of the hand is given; note that it can be either a grasp or pre-grasp configuration, which can be obtained with different approaches (e.g. Ciocarlie and Allen 2009; Roa and Suárez 2009). The result of the proposed planner is compared with the case where no PMDs are used, i.e. samples of the hand are obtained from the whole hand configuration space.

The quantitative results for the planning approach are summarized and compared in Table 2, that show the values obtained for 100 runs. These results were obtained using a desktop computer equipped with a $3.00 \mathrm{GHz}$ Intel
Table 2 Comparison in averaged values (over 100 runs) between the proposed approach with the hand workspace reduced using PMDs and the case with the search space equal to the full configuration space

\begin{tabular}{lll}
\hline Type of search space considered & Reduced & Full \\
\hline Time to find a solution [s] & 10.39 & 915.28 \\
Smoothing time [s] & 0.096 & 15.20 \\
Final neighboring threshold $\left(\rho_{a}\right)$ & 0.0013 & 0.0631 \\
Maximum num. of trials $(j \times h$ loops) & 22.55 & 270.44 \\
Total num. of samples $($ numSamples $)$ & 698.82 & 7274.78 \\
Total num. of nodes in the PRM & 29.18 & 567.22 \\
$\%$ of PRM nodes generated with: & & \\
$\quad$ thumb-base + 1 PMDs & $22.4 \%$ & N/A \\
$\quad$ thumb-base + 2 PMDs & $26.5 \%$ & N/A \\
thumb-base + 3 PMDs & $16.5 \%$ & N/A \\
thumb-base + 4 PMDs & $34.7 \%$ & N/A \\
Total nodes in the solution path & 3.66 & 3.22 \\
\hline
\end{tabular}

Table 3 Parameters of the planner (the values shown for the adaptive parameters are the initial ones). The values in parenthesis are used when no PMDs are considered

\begin{tabular}{lllll}
\hline$\rho_{a}$ & $K$ & $N$ & $n_{A}$ & $n_{H}$ \\
\hline 0.001 & 10 & $100(10,000)$ & $10(20)$ & $10(20)$ \\
\hline
\end{tabular}

Core 2 CPU, running Windows operating system and using the planner parameters shown in Table 3. The maximum number $N$ of samples was chosen large enough to allow finding a solution in all cases, i.e. no failure runs happened.

The results show a noticeable decrease in the number of samples required when using PMDs (less than the $10 \%$ of the samples required without using PMDs), and also a decrease in computational time (more than 80 times faster). This is basically due to the fact that using PMDs collisionfree samples are more easily found. The maximum number of PMDs needed to solve this task was 4 in all the runs, i.e. the difficult parts of the path always required samples of a 5-dimensional subspace $\mathcal{S} C^{\mathrm{h}}$ (generated by the thumbbase and the first four PMDs). On the contrary, parts of the path farther from the obstacles were sampled with lowerdimensional subspaces. The mean percentage of PRM nodes generated with 2, 3, 4 and 5-dimensional subspaces is reported in the table. The difficult parts of the path also required more trials to obtain free hand-arm configurations, i.e. more passes within the $h$ and $j$ for-loops of the main algorithm. Using PMDs the mean maximum number of trials was smaller (less than $10 \%$ of the required without using PMDs). Also, since using PMDs required less passes of the algorithm, the final arm search space (determined by $\rho_{a}$ ) was smaller than without using PMDs, resulting in 


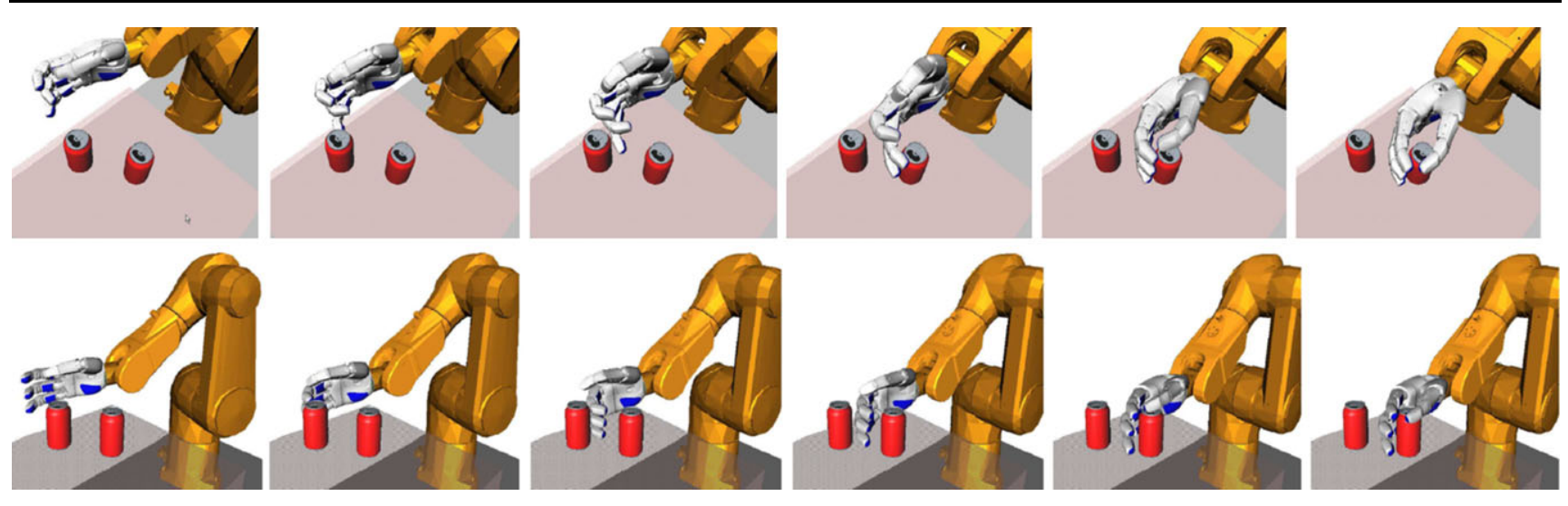

Fig. 14 Qualitative comparison between the approach that considers the full hand configuration space (top) and the proposed approach that reduces the hand workspace using the PMDs (bottom). The use of PMDs resulted in a path composed of a smooth sequence of human-like postures

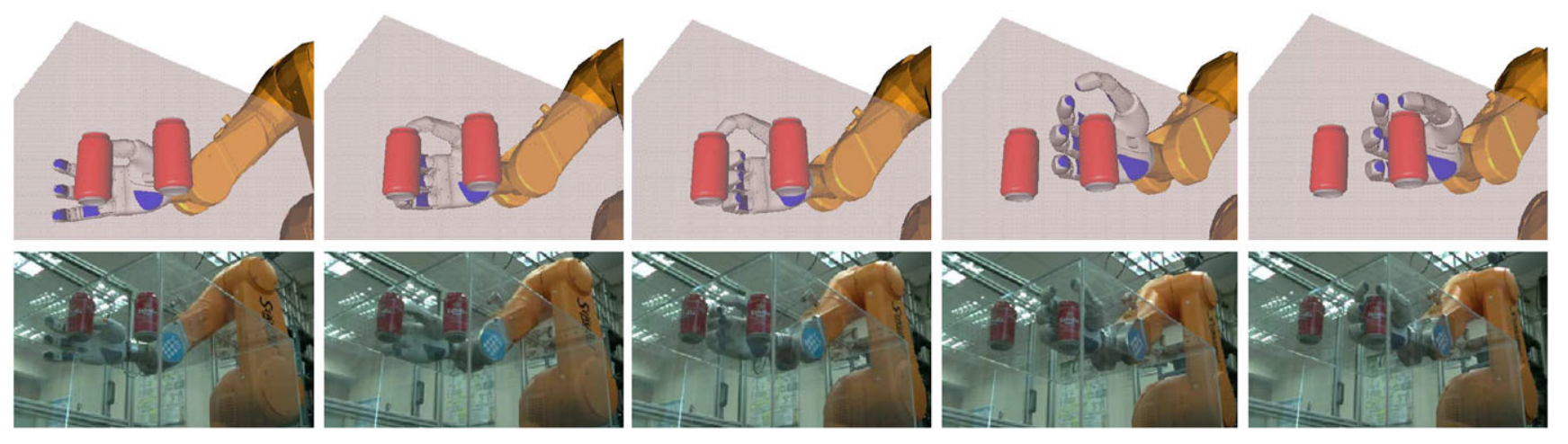

Fig. 15 Simulation of a solution path and real execution in the actual hand-arm system described in Sect. 3

paths more close to the rectilinear segment connecting $\boldsymbol{c}_{\text {ini }}$ and $\boldsymbol{c}_{\text {goal }}$.

The qualitative results are also interesting (see Fig. 14). Using PMDs the solution path resembles a sequence of human-like postures, while the solution found when sampling the whole hand configuration space contains awkward hand postures, even though a smoothing procedure is always applied (described above in function FINDPATH).

As it was previously mentioned, a solution path was successfully implemented on the actual hand-arm system described in Sect. 3. Figure 15 shows the screenshots of both the virtual and the real path at their equivalent points on the path (see also the accompanying video for a continuous depiction of the example). The implementation on the real hand-arm system makes visible the usefulness of the proposed algorithms.

\subsection{Performance study}

Assuming a given grasp or pre-grasp configuration, the proposed approach looks for the final approaching motion, where the collisions are more likely to occur with the hand rather than with the arm (i.e. collision-free solution paths

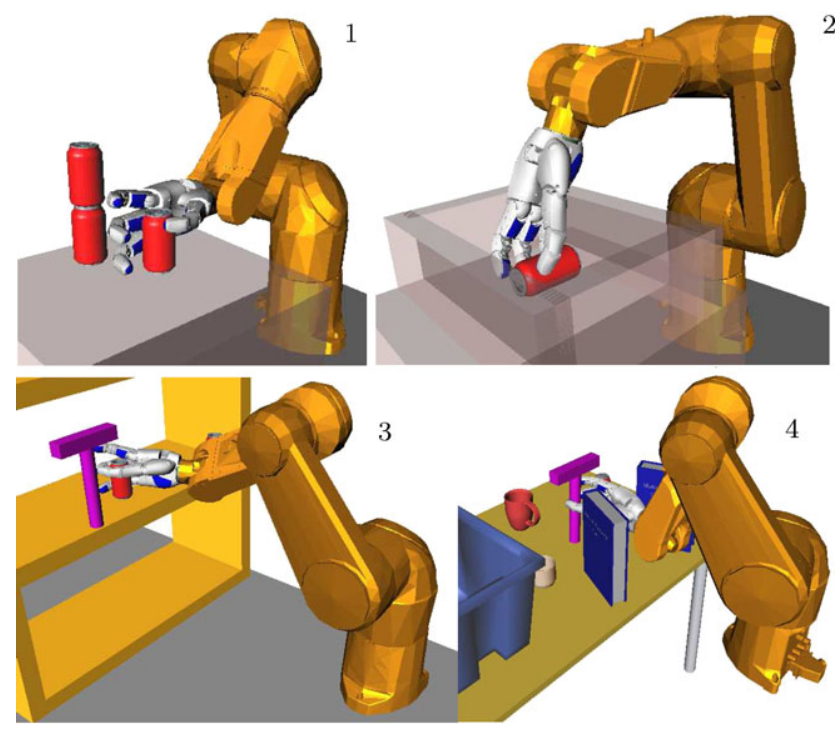

Fig. 16 Goal configurations of the hand-arm system for some of the tasks used to test the planner: (1) Cans on a desk; (2) Can in a box; (3) Cans in a shelf; (4) T-shape object in a complex scene

will require finger motions and only slight arm deviations from the straight motion). With this in mind, the planner has 


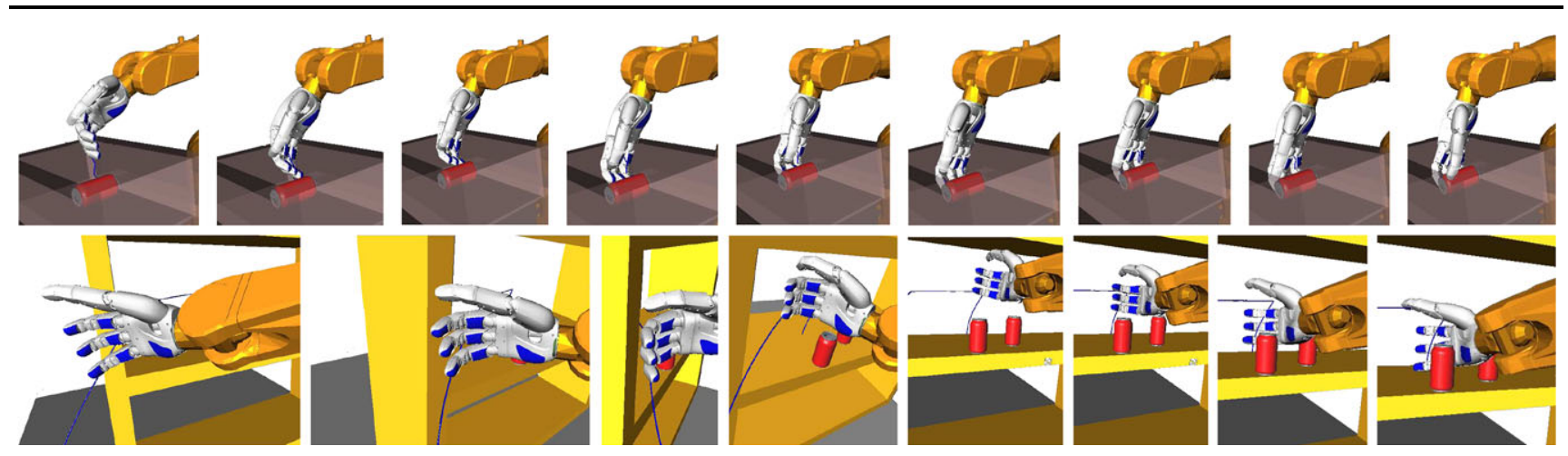

Fig. 17 Some screen shots of the solution paths of two tasks. Note how the motion of the fingers avoid collisions

Table 4 Comparison of the performance of the planner used to solve different problems. These values are the means from 1000 runs. Times in seconds

\begin{tabular}{lrrrr}
\hline Problem & \multicolumn{1}{c}{1} & 2 & \multicolumn{1}{c}{3} & \multicolumn{1}{c}{4} \\
\hline Time to find a solution & 21.06 & 7.75 & 71.64 & 24.09 \\
Smoothing time & 0.124 & 0.022 & 0.821 & 0.142 \\
& & & & \\
Number of samples & 1188.7 & 437.7 & 2093.8 & 2205.6 \\
Nodes in the PRM & 53.6 & 24.6 & 131.7 & 81.9 \\
Nodes in solution path & 3.9 & 3.4 & 4.2 & 4.9 \\
\hline
\end{tabular}

been evaluated on several problems, four of them shown in Fig. 16, with different degrees of difficulty. In comparison with the task of Sect. 6.2: (a) the task in Fig. 16-1 has a narrower passage; (b) the task in Fig. 16-2 has the goal configuration closer to the obstacles; (c) the task in Fig. 16-3 has the rectilinear path to the goal more obstructed by the presence of the T-shaped object and of the shelf itself; (d) the task in Fig. 16-4 has a more cluttered environment with a longer narrow passage (this task is similar to that used in Berenson et al. (2007)). The solution paths required motions of the finger joints, maintaining the robot configurations as close to the rectilinear path as possible, and resulted in smooth sequences of human-like configurations (Fig. 17). The algorithm was run in a computer with a I5 processor with 4 cores and $4 \mathrm{~Gb}$ of RAM, under Windows 7 64-bit. The testing procedure was parallelized using the MPI library (1) in order to use all cores. The quantitative results are shown in Table 4. Note that the fourth task required the generation of more samples than the third task, since the environment is more cluttered and many samples resulted in collision, but could be solved with a PRM composed of less nodes because the narrow passage was more aligned with the direction connecting $\boldsymbol{c}_{\text {ini }}$ and $\boldsymbol{c}_{\text {goal }}$. Therefore, the time to find a solution was larger in the third task because the validation of the PRM edges is time-consuming.

\section{Discussion}

The value of $\rho_{a}$ determines how far the arm path can be from the rectilinear segment in $\mathcal{C}^{\mathrm{a}}$ that connects $\boldsymbol{c}_{\text {ini }}^{\mathrm{a}}$ and $\boldsymbol{c}_{\text {goal }}^{\mathrm{a}}$. During the final approaching motion to grasp an object, the potential collisions are likely to occur with the hand, not with the arm. Therefore, finger motions are usually required to avoid collisions, although slight arm deviations from the straight motion may be of great help. The value of $\rho_{a}$ also determines the number of samples considered for each pass of the general loop, i.e. the number of hypercubes $B^{\mathrm{a}}$ considered, although the neighboring threshold is an independent value and configurations sampled from non-contiguous hypercubes can be connected in the roadmap. The value of $\rho_{a}$ is iteratively increased, and the initial chosen value is not a very critical issue. It has to be neither too small (since then its increase could be too slow and too many samples could be required), nor to large (since then the search space could be too large and also too many samples could be required). Good results were obtained for different tasks using values of $\rho_{a}$ between 0.001 and 0.05 ( $\rho_{a}$ is given as a nondimensional parameter because the range of each arm joint was normalized to $[0,1])$.

The proposed approach determines the hand postures using as few PMDs as possible, which results in smoother motions all along the solution path. Moreover, the use of PMDs results in a better computational efficiency because the percentage of collision-free samples is much higher than in the case where the finger joints are directly sampled.

The values $n_{H}$ and $n_{A}$ allow several trials in the difficult parts of the path, giving more freedom to find a collisionfree hand-arm configuration. These values are by no means critical, since the successive passes of the main loop also permit the resampling of the difficult areas.

The distance threshold used to consider two configurations as neighboring samples is set equal to the distance between the initial and the goal configuration, it is not a user-defined parameter. In the scope of the final approaching motion to grasp an object, and taking into account that 
the algorithm tightly bounds the search space, this selection allowed to find the connectivity between the initial and the goal configurations using much less samples.

The paths generated over several runs on the same example are obviously different but qualitatively quite similar. The reason is that the approach always starts by sampling within regions of increasing volume centered along the rectilinear path that connects the initial and the goal robot configurations.

\section{Conclusions}

The paper has presented a motion planner for a hand-arm robotic system. The proposal pursues efficiency and humanlikeliness in the hand postures. Human hand workspace is captured using a sensorized glove and mapped to the mechanical hand workspace where the most relevant principal motion directions that capture the (human-like) couplings can be identified using Principal Component Analysis. Both aims can be achieved by considering, for the finger joints, the lower-dimensional subspace determined by the main principal motion directions.

The planner is focused on the final approaching motion to a grasp or pre-grasp configuration. Planning is done with a probabilistic roadmap planner, and the dimensionality reduction in the hand search space results in lower computational times. The proposed approach has no critical parameters to be tuned. The hand search space is iteratively increased in dimension and the arm search space in volume, as much as it is required by the difficulty of the task. The validity of the approach has been demonstrated in both simulations and real experiments.

Acknowledgements The authors would like to thank Jorge A. García for his collaboration in the data acquisition and mapping procedures.

\section{References}

Ali, M. S., Kyriakopoulos, K. J., \& Stephanou, H. E. (1993). The kinematics of the Anthrobot-2 dextrous hand. In Proc. of the IEEE int. conf. on robotics and automation (Vol. 3, pp. 705-710).

Bekey, G. A., Tomovic, R., \& Zeljkovic, I. (1990). Control architecture for the Belgrade/USC hand (pp. 136-149). New York: Springer.

Berenson, D., Diankov, R., Nishiwaki, K., Kagami, S., \& Kuffner, J. (2007). Grasp planning in complex scenes. In Proc. of the IEEE$R A S$ international conference on humanoid robots.

Berenson, D., Srinivasa, S., Ferguson, D., \& Kuffner, J. (2009). Manipulation planning on constraint manifolds. In Proc. of the IEEE int. conf. on robotics and automation (pp. 625-632).

Biagiotti, L., Lotti, F., Melchiorri, C., \& Vassura, G. (2004). How far is the human hand? a review on anthropomorphic robotic endeffectors (Tech. rep.). University of Bologna.

Bicchi, A. (2000). Hands for dexterous manipulation and robust grasping: a difficult road toward simplicity. IEEE Transactions on Robotics and Automation, 16(6), 652-662.
Bluethmann, W., Ambrose, R., Diftler, M., Askew, S., Huber, E., Goza, M., Rehnmark, F., Lovchik, C., \& Magruder, D. (2003). Robonaut: a robot designed to work with humans in space. Autonomous Robots, 14(2), 179-197.

Boor, V., Overmars, M. H., \& van der Stappen, A. F. (1999). The Gaussian sampling strategy for probabilistic roadmap planners. In Proc. of the IEEE int. conf. on robotics and automation (pp. 1018-1023).

Butterfass, J., Fischer, M., Grebenstein, M., Haidacher, S., \& Hirzinger, G. (2004). Design and experiences with DLR hand II. In Proc. of the world automation congress (Vol. 15, pp. 105-110).

Caffaz, A., \& Cannata, G. (1998). The design and development of the DIST-Hand dextrous gripper. In Proc. of the IEEE int. conf. on robotics and automation (Vol. 3, pp. 2075-2080).

Cheng, H. L., Hsu, D., Latombe, J. C., \& Sanchez-Ante, G. (2006). Multi-level free space dilation for sampling narrow passages in prm planning. In Proc. of the IEEE int. conf. on robotics and automation (pp. 1255-1260).

Choset, H., Lynch, K. M., Hutchinson, S., Kantor, G., Burgard, W., Kavraki, L. E., \& Thrun, S. (2005). Principles of robot motion. Cambridge: MIT Press.

Ciocarlie, M. T., \& Allen, P. K. (2009). Hand posture subspaces for dexterous robotic grasping. The International Journal of Robotics Research, 28(7), 851-867.

Cortés, J., \& Siméon, T. (2004). Sampling-based motion planning under kinematic loop closure constraints. In Proc. of the 6th int. workshop on the algorithmic foundations of robotics (pp. 59-74).

Gabiccini, M., \& Bicchi, A. (2010). On the role of hand synergies in the optimal choice of grasping forces. In Proc. of robotics: science and systems.

Gazeau, J. P., Zehloul, S., Arsicault, M., \& Lallemand, J. P. (2001). The LMS hand: force and position controls in the aim of the fine manipulation of objects. In Proc. of the IEEE int. conf. on robotics and automation (Vol. 3, pp. 2642-2648).

Geraerts, R., \& Overmars, M. H. (2006). Sampling and node adding in probabilistic roadmap planners. Robotics and Autonomous Systems, 54, 165-173.

Gropp, W., Skjellum, A., Lusk, E. (1999). Using MPI: Portable parallel programming with the message-passing interface. Cambridge: MIT Press.

Halton, J. (1960). On the efficiency of certain quasi-random sequences of points in evaluating multi-dimensional integrals. Numerische Mathematik, 2, 84-90.

Hsu, D., Jiang, T., Reif, J., \& Sun, Z. (2003). The bridge test for sampling narrow passages with probabilistic roadmap planners. In Proc. of the IEEE int. conf. on robotics and automation (pp. 4420-4426).

Hsu, D., Sanchez-Ante, G., \& Sun, Z. (2005). Hybrid PRM sampling with a cost-sensitive adaptive strategy. In Proc. of the IEEE int. conf. on robotics and automation (pp. 3874-3880).

Hsu, D., Latombe, J. C., \& Kurniawati, H. (2006). On the probabilistic foundations of probabilistic roadmap planning. The International Journal of Robotics Research, 25(7), 627-643.

Jacobsen, S. C., Wood, J. E., Knutti, D. F., \& Biggers, K. B. (1984). The UTAH/M.I.T. dextrous hand: work in progress. The International Journal of Robotics Research, 3(4), 21-50.

Jolliffe, I. (2002). Springer series in statistics. Principal component analysis. Upper Saddle River: Springer.

Kavraki, LE, \& Latombe, J. C. (1994). Randomized preprocessing of configuration for fast path planning. In Proc. of the IEEE int. conf. on robotics and automation (pp. 2138-2145).

Kavraki, L. E., Svestka, P., Latombe, J. C., \& Overmars, M. K. (1996). Probabilistic roadmaps for path planning in high-dimensional configuration spaces. IEEE Transactions on Robotics and Automation, 12(4), 566-580. 
Kawasaki, H., Komatsu, T., \& Uchiyama, K. (2002). Dexterous anthropomorphic robot hand with distributed tactile sensor: Gifu hand II. IEEE/ASME Transactions on Mechatronics, 7(3), 296-303.

Kuffner, J. J., \& LaValle, S. M. (2000). RRT-connect: an efficient approach to single-query path planning. In Proc. of the IEEE int. conf. on robotics and automation (pp. 995-1001).

Kuffner, J. J., Kagami, S., Nishiwaki, K., Inaba, M., \& Inoue, H. (2002). Dynamically-stable motion planning for humanoid robots. Autonomous Robots, 12(1), 285-300.

Kuipers, L., \& Niederreiter, H. (2005). Uniform distribution of sequences. New York: Dover.

Kurniawati, H., \& Hsu, D. (2006). Workspace-based connectivity oracle: an adaptive sampling strategy for PRM planning. In S. Akella et al. (Eds.), Algorithmic foundations of robotics VII. Berlin: Springer.

Lin, L. R., \& Huang, H. P. (1996). Mechanism design of a new multifingered robot hand. In Proc. of the IEEE int. conf. on robotics and automation (Vol. 2, pp. 1471-1476).

Lotti, F., Tiezzi, P., Vassura, G., Biagiotti, L., Palli, G., \& Melchiorri, C. (2005). Development of UB hand 3: early results. In Proc. of the IEEE int. conf. on robotics and automation (pp. 4488-4493).

Lovchik, C. S., Diftler, M. A. (1999). The robonaut hand: a dexterous robot hand for space. In Proc. of the IEEE int. conf. on robotics and automation (Vol. 2, pp. 907-912).

Lozano-Perez, T. (1987). A simple motion-planning algorithm for general robot manipulators. IEEE Journal of Robotics and Automation, 3(3), 224-238.

Murrieta-Cid, R., Tovar, B., \& Hutchinson, S. (2005). A samplingbased motion planning approach to maintain visibility of unpredictable targets. Autonomous Robots, 19(3), 285-300.

Peña, E., Yang, J., \& Abdel-Malek, K. (2005). Santos ${ }^{\text {TM }}$ hand: a 25degree-of-freedom model. In Proc. of SAE digital human modeling for design and engineering, Iowa City, Iowa, USA.

Pérez, A., \& Rosell, J. (2009). A roadmap to robot motion planning software development. Computer Applications in Engineering Education. doi:101002/cae20269.

Roa, M., \& Suárez, R. (2009). Finding locally optimum force-closure grasps. Robotics and Computer-Integrated Manufacturing, 25(3), 536-544.

Rodríguez, A., Pérez, A., Rosell, J., \& Basañez, L. (2009). Samplingbased path planning for geometrically-constrained objects. In Proc. of the IEEE int. conf. on robotics and automation (pp. 20742079).

Rosales, C., Ros, L., Porta, J. M., \& Suárez, R. (2011). Synthesizing grasp configurations with specified contact regions. The International Journal of Robotics Research, 30(4), 431-443.

Rosell, J., Sierra, X., Palomo, L., \& Suárez, R. (2005). Finding grasping configurations of a dexterous hand and an industrial robot. In Proc. of the IEEE int. conf. on robotics and automation (pp. 1178-1183).

Rosell, J., Roa, M., Pérez, A., \& García, F. (2007). A general deterministic sequence for sampling d-dimensional configuration spaces. Journal of Intelligent and Robotic Systems, 50(4), 361-374.

Rosell, J., Suárez, R., Rosales, C., García, J. A., \& Pérez, A. (2009). Motion planning for high DOF anthropomorphic hands. In Proc. of the IEEE int. conf. on robotics and automation (pp. 40254030).

Safonova, A., Hodgins, J. K., \& Pollard, N. S. (2004). Synthesizing physically realistic human motion in low-dimensional behaviorspecific spaces. ACM Transactions on Graphics, 23(3), 514-521.

Saha, M., Latombe, J. C., Chang, Y. C., \& Prinz, F. (2005). Finding narrow passages with probabilistic roadmaps: the small-step retraction method. Autonomous Robots, 19(3), 301-319.

Santello, M., Flanders, M., \& Soechting, J. F. (1998). Postural hand synergies for tool use. Journal of Neuroscience, 18(23), 10,10510,115 .
Schunk GmbH \& Co KG (2006). Schunk anthropomorphic hand. http://www.schunk.com/.

Shadow Robot Company (2003). Design of a dextrous hand for advanced clawar applications. In Climbing and walking robots and the supporting technologies for mobile machines (pp. 691-698).

Stilman, M. (2010). Global manipulation planning in robot joint space with task constraints. IEEE Transactions on Robotics, 26(3), 576584.

Suárez, R., \& Grosch, P. (2005). Mechanical hand MA-I as experimental system for grasping and manipulation. In VideoProc. of the IEEE int. conf. on robotics and automation, Barcelona.

Suárez, R., Rosell, J., Pérez, A., \& Rosales, C. (2009). Efficient search of obstacle-free paths for anthropomorphic hands. In Proc. of the IEEE/RSJ int. conf. on intelligent robots and systems (pp. 17731778).

Tsoli, A., \& Jenkins, O. C. (2007). 2D subspaces for user-driven robot grasping. In Proc. of the RSS 2007 workshop on robot manipulation: sensing and adapting to the real world.

van der Berg, J. P., \& Overmars, M. H. (2005). Using workspace information as a guide to non-uniform sampling in probabilistic roadmap planners. The International Journal of Robotics Research, 24(12), 1055-1071. 
